\title{
EXCESS HEAT PRODUCTION IN Pd/D DURING PERIODIC PULSE DISCHARGE CURRENT IN VARIOUS CONDITIONS
}

\author{
A. B. Karabut \\ FSUE "LUCH"
}

24 Zheleznodorozhnaya St, Podolsk, Moscow Region, 142100, Russia.

\begin{abstract}
Experimental data from low energy nuclear reactions (LERN) in condensed media are presented. The nuclear reactions products were found in solid cathode media used in glow discharge. Apparently, the nuclear reactions were initiated when bombarding the cathode surface by plasma ions with the energy of 1.0-2.0 keV. Excess heat from a high current glow discharge reaction in $\mathrm{D}_{2}$, Xe and $\mathrm{Kr}$ using cathodes already charged with preliminary deuterium-charged $\mathrm{Pd}$ and Ti cathode samples are given. Excess heat up to $10-15 \mathrm{~W}$ and efficiency up to $130 \%$ was recorded under the experiments for Pd cathode samples in $\mathrm{D}_{2}$ discharge. Excess heat up to $5 \mathrm{~W}$ and efficiency up to $150 \%$ was recorded for Pd cathodes that were charged with deuterium before the run, in $\mathrm{Xe}$ and $\mathrm{Kr}$ discharges. At the same time excess heat was not observed for pure Pd cathode samples in $\mathrm{Xe}$ and $\mathrm{Kr}$ discharges. The formation of impurity nuclides $\left({ }^{7} \mathrm{Li},{ }^{13} \mathrm{C},{ }^{15} \mathrm{~N},{ }^{20} \mathrm{Ne},{ }^{29} \mathrm{Si},{ }^{44} \mathrm{Ca},{ }^{48} \mathrm{Ca}\right.$, $\left.{ }^{56} \mathrm{Fe},{ }^{57} \mathrm{Fe},{ }^{59} \mathrm{Co},{ }^{64} \mathrm{Zn},{ }^{66} \mathrm{Zn},{ }^{75} \mathrm{As},{ }^{107} \mathrm{Ag},{ }^{109} \mathrm{Ag},{ }^{110} \mathrm{Cg},{ }^{111} \mathrm{Cg},{ }^{112} \mathrm{Cg},{ }^{114} \mathrm{Cg},{ }^{115} \mathrm{In}\right)$ with the efficiency up to $10{ }^{13}$ atoms/s was recorded. The isotopic ratios of these new nuclides was quite different from the natural ratios. Soft X-ray radiation from the solid-state cathode with the intensity up to $0.01 \mathrm{~Gy} / \mathrm{s}$ was recorded in experiments with discharges in $\mathrm{H}_{2}, \mathrm{D}_{2}, \mathrm{Ar}$, Xe and $\mathrm{Kr}$. The X-ray radiation was observed in bursts of up to $10^{6}$ photons, with up to $10^{5}$ bursts per second while the discharge was formed and within $100 \mathrm{~ms}$ after turning off the discharge current. The results of the X-ray radiation registration showed that the exited energy levels have a lifetime up to $100 \mathrm{~ms}$ or more, and the energy of $1.2-2.5 \mathrm{keV}$. A possible mechanism for producing excess heat and nuclear transmutation reactions in the solid medium with the exited energy levels is considered.
\end{abstract}

\section{Introduction}

We have conducted experimental research on low energy nuclear reactions (LENR). By LENR we mean nuclear reactions initiated by a low energy action (from units up to a thousand of eV) in condensed media. Under such a low energy action the non-equilibrium energy states with a temperature up to $3 \mathrm{keV}$ and lifetimes up to tens of milliseconds can be formed in the condensed medium. Occurrence of such states was found during the experiments when registering X-rays with energy up to $3 \mathrm{keV}$. Hypothetically, X-ray emission and other accompanying effects indicate a fundamentally new physical phenomenon unknown before: metastable long-living (up to tens of $\mathrm{ms}$ ) states with the excitation energy of $1-2 \mathrm{keV}$ and more are formed in the crystal solid lattice within the solid when bombarding its surface by plasma ions of an electrical discharge . Therefore, $\mathrm{L}, \mathrm{M}$ excited energy states with the occupation density $\mathrm{n}_{\mathrm{v}-\mathrm{d}}\left(\mathrm{cm}^{-3}\right)$ and characteristic temperature $\mathrm{T}_{\mathrm{L}, \mathrm{M}} \approx 1-2 \mathrm{keV}$ and more $\left(20,000,000{ }^{\circ} \mathrm{K}\right)$ are formed in the solid after each pass of the current glow discharge pulse. These power conditions exist for the characteristic time $\tau_{\mathrm{L}, \mathrm{M}}$ (up to $100 \mathrm{~ms}$ and more). Realization of nuclear reactions of transmutation with evolving heat power and accumulation of impurity elements within the cathode material is possible in such medium. The probability of such reactions proceeding is defined by the characteristic temperature, excitation energy level density, and lifetime of excited levels. These nuclear reactions (LENR) can be called non-equilibrium nuclear reactions.

The experimental measurements of the excess heat yield, anomalous isotopes in the cathode material, heavy particle emission and soft X-rays at large densities of the discharge current were carried out with a device that underwent high-current glow discharge for a long time. As applied to developing a long operating reactor for the heat power production, the research was carried out on the modes with small density of discharge current, to finding a possible mechanism of initiating non-equilibrium nuclear transmutation reactions in the solid medium of the cathode sample. 


\section{MEASUREMENT OF EXCESS HEAT BY A FLOW CALORIMETER}

The measurements were carried out using the glow discharge device consisting of a water-cooled vacuum chamber, cathode and anode assemblies. The cathode design allowed placing the cathode samples made of various materials on the cooled surface. The device consisted of three units: the cathode, anode and chamber had independent channels of water cooling. Each cooling channel included two thermocouples at the inlet and outlet, and a flowmeter. The device was placed into a thermal insulating package (Fig. 1) and was a flow calorimeter.

Non-deuterium-charged $\mathrm{Pd}$ cathode samples in $\mathrm{Xe}$ and $\mathrm{Kr}$ discharges were used in the tests. In contrast to the experiments carried out before, the mode of "plasma anode" was used. The anode was set out at the chamber wall (Fig. 1) and was put into the plasma area being above the cathode. The pulse-periodic electrical power supply was used. The electrical parameters: discharge current and voltage were recorded using a twochannel computer digital oscillograph. The electrical power was determined according to the expression

$$
\mathrm{P}_{\mathrm{el}}=1 / \mathrm{T} \times \int \mathrm{U}(\mathrm{t}) \mathrm{I}(\mathrm{t}) \mathrm{dt} \text {. }
$$

When excess heat was released inside the chamber $\mathrm{P}_{\mathrm{EH}}$, its value could be determined by

$$
\mathrm{P}_{\mathrm{EH}}=\left(\mathrm{P}_{\mathrm{HC}}+\mathrm{P}_{\mathrm{HA}}+\mathrm{P}_{\mathrm{HCh}}\right)-\mathrm{P}_{\mathrm{el}} \pm \Delta \mathrm{P}_{\mathrm{error}}
$$

where $\mathrm{P}_{\mathrm{el}}$ - input electrical power of the glow discharge, $\mathrm{P}_{\mathrm{HC}}, \mathrm{P}_{\mathrm{HA}}, \mathrm{P}_{\mathrm{HCh}}$ - output heat power by the cooling water of the cathode, anode and chamber respectively, $\Delta \mathrm{P}_{\text {error }}-$ complete absolute error of the power measurement for given measuring system, $\mathrm{T}$-period of following the pulses of the glow discharge current. The measurements system allowed to record the electrical power input into the discharge and heat capacity output by the cooling water with accuracy of $0.6 \mathrm{~W}$ at the absolute value of the electrical power up to $120 \mathrm{~W}$ (Relative error $\pm 0.5 \%$,).

Two typical operating modes of the device were used in the experiments. High density discharge current (more than $20 \mathrm{~mA} / \mathrm{cm}^{2}$ ) was used in the first set of experiments. Under these modes deuterium loading into Pd cathode samples did not take place. The absolute excess power had a large value (up to $20 \mathrm{~W}$ ) but total efficiency coefficient was less than $130 \%$ (Fig. 2). In the other set of experiments the current density did not exceed $100 \mathrm{~mA} / \mathrm{cm}^{2}$. Under such values of the discharge current in $\mathrm{D}_{2}$, continuous loading of $\mathrm{D}_{2}$ into Pd ran up to saturation. The experiments were carried out with $\mathrm{Pd}$ cathode samples in $\mathrm{D}_{2}$ discharge, with the preliminary deuterium-charged $\mathrm{Pd}$ cathode samples in $\mathrm{Xe}$ and $\mathrm{Kr}$ discharges. The amount of $\mathrm{D}_{2}$ loaded into the cathode was determined by reducing the pressure in the chamber. $\mathrm{D}_{2}$ was periodically supplied into the chamber for maintaining the required pressure. Deuterium loading was determined by the volume of the gas absorbed from the discharge chamber. When high loading was achieved, the D/Pd ratio was close to 1 .

The heat measurements were carried out for Pd cathode samples in glow discharge when changing the following parameters: discharge current density, voltage, duration of current pulses and time period between the current pulses of the power supply. The absolute value of the excess heat power and thermal efficiency grew with increasing power input into the discharge (Fig. 3). The maximum excess heat power and thermal efficiency were recorded under the following conditions: while loading D into Pd took place, or when D left $\mathrm{Pd}$; and when the time period between the current pulses was much greater than the duration of the discharge current pulses.

This result showed that the excess heat continued in the cathode sample after the current was turned off (during the time period between the current pulses). The maximum value of the excess heat was recorded at the discharge burning voltage of $1000-1400 \mathrm{~V}$.

Large values for excess heat power and thermal efficiency were recorded for previously deuteriumcharged cathode samples in Xe and Kr discharges (Fig. 4). Two typical groups of the results can be noted: relatively large values of the excess heat power and efficiency coefficient (curve 1) and the group of results with lower values for excess heat power efficiency coefficient (curve 2). The large values of the excess heat power and efficiency coefficient were observed when partial loading of deuterium into Pd cathode samples took place, or when deuterium left Pd cathode samples. Excess heat was not produced using cathodes made of pure $\mathrm{Pd}$ (not deuterium-charged) in $\mathrm{Xe}$ and $\mathrm{Kr}$ discharges (Fig. 5). Thus, it was experimentally shown that excess heat production was defined by two processes: 1 - deuterium should be loaded into the medium of the crystal solid lattice. 2 - the crystal lattice should obtain initial excitation; high-energy long-living exited levels should be created in the solid. These exited conditions could be created by an additional source (for example, by a flow of inert gas ions) 
The three-channel system of separate recording of the output heat power (anode, cathode, and chamber) allowed us to define the structure of the excess heat during glow discharge. As Fig. 6 and 7 show, the high efficiency values were recorded in the experiments with the large relative heat release on the cathode. These results show that the excess heat was released mainly on the cathode.

\section{REGISTRATION OF ANOMALOUS NUCLIDES}

The analysis of impurities and or possible anomalous nuclides in the cathode sample material before and after the experiments when using the device of the high-current glow discharge [1] was made, assuming that the recorded excess heat [1] was caused by nuclear reactions [2]. The following methods were used: spark mass spectrometry, secondary ionic mass spectrometry, and secondary neutral mass spectrometry. Difference in the content of the anomalous elements before and after the experiment was defined as storage of the elements during the experiment.

The secondary ion mass spectrometry (SIMS) analysis included the following operations: removal the upper $1.5 \mathrm{~nm}$ layer of the cathode surface by plasma etching; scanning the first and second layers down $5 \mathrm{~nm}$ to determine the content of any anomalous nuclides that might be present, removal another layer $700 \mathrm{~nm}$ thick and repeating the scan of the third and fourth layers to a $5 \mathrm{~nm}$ depth (Fig. 8).

Anomalous or impurity elements with the mass approximately half as large as $\mathrm{Pd}$ and with mass close to $\mathrm{Pd}$ mass were recorded in the near-surface layer having the thickness of $100 \mathrm{~nm}$ in amounts up to several dozen percent. The main elements (those with more than $1 \%$ of the content) were ${ }^{7} \mathrm{Li},{ }^{12} \mathrm{C},{ }^{15} \mathrm{~N},{ }^{20} \mathrm{Ne},{ }^{29} \mathrm{Si}$, ${ }^{44} \mathrm{Ca},{ }^{48} \mathrm{Ca},{ }^{56} \mathrm{Fe},{ }^{57} \mathrm{Fe},{ }^{59} \mathrm{Co},{ }^{64} \mathrm{Zn},{ }^{66} \mathrm{Zn},{ }^{75} \mathrm{As},{ }^{107} \mathrm{Ag},{ }^{109} \mathrm{Ag},{ }^{110} \mathrm{Cg},{ }^{111} \mathrm{Cg},{ }^{112} \mathrm{Cg},{ }^{114} \mathrm{Cg},{ }^{115} \mathrm{In}$ (table 1). The impurity content in the cathode bulk sample at different depths was defined. The content at the depth of 800 $\mathrm{nm}$ decreased by 1.5 -2 times in comparison with the near-surface layers (Fig. 9, table 1).

The results of these measurements show that production of the anomalous nuclides occurred in the sample material at depths of up to $1000 \mathrm{~nm}$ (up to 4000 atom layers) from the cathode surface.

Table 1.

\begin{tabular}{|l|l|l|l|l|}
\hline $\begin{array}{l}\text { A } \\
\text { Anom. } \\
\text { nuclide }\end{array}$ & $\begin{array}{l}1 \text { scan } \\
10 \mathrm{~nm}, \\
\text { content, } \\
\%\end{array}$ & $\begin{array}{l}2 \text { scan } \\
50 \mathrm{~nm}, \\
\text { content, } \\
\%\end{array}$ & $\begin{array}{l}3 \text { scan } \\
700 \mathrm{~nm}, \\
\text { content, } \\
\%\end{array}$ & $\begin{array}{l}\text { 4 scan } \\
800 \mathrm{~nm}, \\
\text { content, } \\
\%\end{array}$ \\
\hline${ }^{6} \mathrm{Li}$ & 0.075 & 0.22 & 0.21 & 0.16 \\
\hline${ }^{7} \mathrm{Li}$ & 0.84 & 0.53 & 0.45 & 0.47 \\
\hline${ }^{11} \mathrm{~B}$ & 0.14 & 0.31 & 0.18 & 0.18 \\
\hline${ }^{12} \mathrm{C}$ & 0.93 & 0.63 & 0.47 & 0.54 \\
\hline${ }^{13} \mathrm{C}$ & 0.19 & 0.15 & 0.05 & 0.06 \\
\hline${ }^{20} \mathrm{Ne}$ & 0.14 & 0.27 & 0.14 & 0.16 \\
\hline${ }^{42} \mathrm{Ca}$ & 0.72 & 1.14 & 1.08 & 0.8 \\
\hline${ }^{44} \mathrm{Ca}$ & 2.0 & 3.2 & 3.1 & 2.6 \\
\hline${ }^{45} \mathrm{Sc}$ & 0.74 & 0.91 & 0.86 & 0.8 \\
\hline${ }^{46} \mathrm{Ti}$ & 0.57 & 0.72 & 0.52 & 0.7 \\
\hline${ }^{47} \mathrm{Ti}$ & 0.25 & 0.14 & 0.31 & 0.14 \\
\hline${ }^{48} \mathrm{Ti}$ & 1.1 & 1.23 & 1.1 & 0.66 \\
\hline${ }^{52} \mathrm{Cr}$ & 0.62 & 0.41 & 0.31 & 0.1 \\
\hline${ }^{56} \mathrm{Fe}$ & 2.9 & 2.6 & 3.1 & 2.7 \\
\hline${ }^{57} \mathrm{Fe}$ & 5.5 & 3.25 & 3.53 & 3.16 \\
\hline${ }^{59} \mathrm{Co}$ & 1.0 & 1.0 & 1.4 & 1.5 \\
\hline${ }^{66} \mathrm{Zn}$ & 0.21 & 0.43 & 0.54 & 1.0 \\
\hline
\end{tabular}

\begin{tabular}{|c|c|c|c|c|}
\hline $\begin{array}{l}\text { A } \\
\text { Anom. } \\
\text { nuclide }\end{array}$ & $\begin{array}{l}1 \text { scan } \\
10 \mathrm{~nm}, \\
\text { content, } \\
\%\end{array}$ & $\begin{array}{l}2 \text { scan } \\
50 \mathrm{~nm}, \\
\text { content, } \\
\%\end{array}$ & $\begin{array}{l}3 \text { scan } \\
700 \mathrm{~nm} \text {, } \\
\text { content, } \\
\%\end{array}$ & $\begin{array}{l}4 \text { scan } \\
800 \mathrm{~nm} \text {, } \\
\text { content, } \\
\%\end{array}$ \\
\hline${ }^{71} \mathrm{Ga}$ & 4.0 & 4.9 & 5.6 & 3.4 \\
\hline${ }^{72} \mathrm{Ge}$ & 5.1 & 4.4 & 5.1 & 6.0 \\
\hline${ }^{75} \mathrm{As}$ & 6.2 & 4.9 & 7.4 & 4.7 \\
\hline${ }^{77} \mathrm{Se}$ & 3.4 & 3.9 & 4.8 & 4.0 \\
\hline${ }^{78} \mathrm{Se}$ & 4.5 & 3.45 & 5.8 & 1.4 \\
\hline${ }^{79} \mathrm{Br}$ & 3.0 & 2.4 & 2.8 & 2.3 \\
\hline${ }^{80} \mathrm{Se}$ & 4.0 & 3.4 & 2.5 & 2.3 \\
\hline${ }^{82} \mathrm{Se}$ & 3.4 & 3.0 & 2.8 & 3.2 \\
\hline${ }^{85} \mathrm{Rb}$ & 2.2 & 3.4 & 3.3 & 3.6 \\
\hline${ }^{88} \mathrm{Sr}$ & 3.1 & 4.4 & 4.2 & 6.0 \\
\hline${ }^{90} \mathrm{Zr}$ & 2.4 & 1.5 & 2.3 & 5.8 \\
\hline${ }^{111} \mathrm{Cd}$ & 2.8 & 3.0 & 3.0 & 3.4 \\
\hline${ }^{112} \mathrm{Cd}$ & 3.4 & 3.2 & 4.2 & 4.5 \\
\hline${ }^{113} \mathrm{Cd}$ & 4.0 & 1.8 & 2.8 & 5.1 \\
\hline${ }^{114} \mathrm{Cd}$ & 4.7 & 3.9 & 3.3 & 3.6 \\
\hline${ }^{115} \mathrm{In}$ & 2.2 & 2.5 & 2.3 & \\
\hline
\end{tabular}

\section{X-RAY REGISTRATION}

Intensive X-ray emission from the solid medium of the cathode samples was recorded in the experiments. The recording of the X-rays was carried out using thermoluminiscent detectors (TLD), X-ray film, and scintillator detectors with photomultipliers [1].

The thermoluminiscent detectors (TLD) on the base of $\mathrm{Al}_{2} \mathrm{O}_{3}$ crystal recorded the values of penetrating radiation, starting from the background values of the environment radiation. These were used to measure the intensity and evaluating the average energy of the soft X-ray emission from the cathode. The detectors are in the form of disks with a diameter of $5 \mathrm{~mm}$ and thickness of $1 \mathrm{~mm}$, enclosed by beryllium foil of various 
thickness $(15 \mu \mathrm{m}, 30 \mu \mathrm{m}, 60 \mu \mathrm{m}, 105 \mu \mathrm{m}, 165 \mu \mathrm{m}, 225 \mu \mathrm{m}$ and $300 \mu \mathrm{m})$ were arranged above the cathode in the special cassette (seven-channel spectrometer). The evaluation of the X-ray energy was made according to changing the radiation dose adsorbed by the TLD detectors provided with Be shields of various thickness.

The radiation intensity (dose power) was defined as the radiation dose absorbed by the detector divided by the experiment time. This expression, and a dependence chart of lg thickness of the half absorption on lg energy of X-rays for beryllium, were used to evaluate the X-ray energy. The values of $1 \mathrm{~g}$ thickness of the half absorption from $1 g$ energy of X-rays for beryllium were taken from [2], Appendix G.

The radiation dose absorbed by the TLD detectors was reduced by an order of magnitude in the $300 \mu \mathrm{m} \mathrm{Be}$ foil compared to the $15 \mu \mathrm{m}$ foil. (Fig 10) The main component of X-ray energy was in the range of $1.0-$ $1.5 \mathrm{keV}$. The value of the $\mathrm{X}$-ray energy determined experimentally increased from 1.2 to $1.5 \mathrm{keV}$ when increasing the thickness of the Be shield from $15 \mu \mathrm{m}$ to $300 \mu \mathrm{m}$ (Fig. 11). It could be assumed that X-rays was emitted from the bulk of the solid-state cathode medium. The part of the radiation from the deeper layers lost initial energy when passing the cathode material. In this case the energy radiation spectrum was displaced to the side of reduced energy. The initial X-rays energy was evaluated as $1.5-2.5 \mathrm{keV}$. The time Xrays characteristics were studied using the scintillator detectors with the photomultipliers [1]. These measurements showed that X-rays emission was observed as a lot of bursts up to $10^{9}$ photons in a burst. The single bursts were recorded after turning off the discharge current within $85 \mathrm{~ms}$ (Fig 12).

\section{DISCUSSION}

The experiments results with the high-current glow discharge carried out for several years to allow allocating the basic processes and conditions of their running.

1 -Production of the excess heat. Excess heat was produced in the bulk of the solid-state medium of the cathode sample under the following conditions:

- - Deuterium should be loaded into the solid-state cathode medium.

- - Initiating excitation of the energy levels of the crystal lattice of the cathode material was necessary.

- - This initiation could be carried out from by a foreign source (for example, by a flow of inert gas ions).

- - The production of the excess heat occurred mainly in the near-surface layer of the cathode sample with the thickness up to $1 \mu \mathrm{m}$ (by the results of recording the impurity nuclides). The volume density of the excess heat had a value up to $10^{5} \mathrm{~W} / \mathrm{cm}^{3}$.

2- Production of the elements isotopes as an impurity to the basic cathode material.

- - The production of the impurity nuclides occurred in the bulk of the solid-state cathode medium presumably as a result of the nuclear transmutation reactions.

- The emission of high-energy heavy ions was not recorded under the experiment. From this fact it was possible to assume, that the nuclear reactions energy was released not as a kinetic energy of the formed impurity nuclides. The impurity nuclides were presumably formed as nuclear isomers (nucleus being in the excited state). From the results of the experiment it followed, that the relaxation of these excited nuclear levels through the gamma- radiation channel was strongly suppressed.

3 - Excitation of the energy levels of the solid-state cathode medium.

- - Formation of the excited energy levels of the crystal lattice was determined by recording the X-rays from solid-state cathode.

- -The X-rays was observed as the bursts of small time duration (presumably up to $10^{-13} \mathrm{~s}$ ). Each burst contained up to $10^{9} \mathrm{x}$-ray quanta with the energy of $1.5-1.8 \mathrm{keV}$. The bursts were recorded in amounts up to $10^{5}$ bursts in a second during the discharge and within $100 \mathrm{~ms}$ after turning off the current.

- - Hypothetically, the mechanism of forming this radiation was as follows. When bombarding the cathode surface by the discharge plasma ions in the solid medium, the excited energy levels with the energy of 1.5 $2.5 \mathrm{keV}$ and lifetime up to $100 \mathrm{~ms}$ were formed. Looking into the concrete physical mechanism of forming these levels calls for additional research. It is possible to assume one of the two possible physical phenomena. 1- Excitation internal L, M electronic shells without ionizing the external electrons. 2- Oscillatory deformation of the electron-nuclear system of the solid ions. The core of electronic shells was displaced from a nucleus with forming a dipole (optical polar phonon).

- - The relaxation of the excited energy levels of the solid medium occurred by emitting the X-rays and, perhaps, fast electrons. 
- - Hypothetically, the relaxation of the excited levels occurred simultaneously from the micro monocrystals making the solid medium. In other words, the totality of the excited ions of the micro monocrystal relaxed simultaneously and gave the X-rays burst.

4- Nuclear transmutation reactions. The excited energy states with the population density of $\mathrm{n}_{\text {exit }}\left(\mathrm{cm}^{-3}\right)$ and characteristic temperature of $\mathrm{T}_{\text {exit }} \approx 1.5-1.8 \mathrm{keV}$ and more (up to $20000000{ }^{\circ} \mathrm{K}$ and more) were formed in the solid after every passing the pulse of the glow discharge current. These energy states existed for the characteristic time $\tau_{\text {exit }}$ (up to $100 \mathrm{~ms}$ and more). Such medium in which the temperature of the crystalline lattice did not exceed some hundreds ${ }^{\circ} \mathrm{K}$ we call a non-equilibrium medium.

- - Non-equilibrium nuclear transmutation reactions are possible in such medium. The probability of running these reactions (and accordingly the value of the excess heat) was determined by criterion: $\mathrm{n}_{\text {exit }} \times \tau_{\text {exit }}>\left(\mathrm{n}_{\text {exit }}\right.$ $\left.\times \tau_{\text {exit }}\right)_{\min }$.

This is a modified Lawson's criterion used to estimate the positive heat output at inertial thermonuclear synthesis.

- - The population density was defined by the parameters of the discharge burning and the cathode sample geometry. The characteristic duration of the existence of excited states was defined by the balance between the processes of the energy levels excitation when passing a pulse of the pumping discharge current and processes of these levels relaxation by emitting the X-rays. Thus, to obtain large quantities of excess heat is necessary to create the high population density of vibration- dipole energy states $\mathrm{n}_{\text {exit }}$ and to suppress the Xrays emission (to increase the lifetime of the excited states $\tau_{\text {exit }}$ ).

5 - The following types of the nuclear transmutation reactions resulting in the formation of the stable nuclides are possible:

$\mathrm{A}+\mathrm{mB} \rightarrow[\mathrm{AB}]^{*}(2)$

$[\mathrm{AB}]^{*} \rightarrow \mathrm{F}^{*} \rightarrow \mathrm{F}+$ Heat $(3)$

$[\mathrm{AB}]^{*} \rightarrow \mathrm{C}^{*}+\mathrm{D}^{*} \rightarrow \mathrm{C}+\mathrm{D}+$ Heat $(4)$

where A - Pd or other element nucleus; B- deuterium or hydrogen; $[\mathrm{AB}]^{*}$ - short-lived intermediate compound nucleus; $\mathrm{m}-1,2,3 \ldots, \mathrm{C}^{*}, \mathrm{D}^{*}$ - nuclear isomers of nuclides with masses less than Pd one; C,D stable nuclides, F - a nuclide with mass more than Pd. First a composite compound-nucleus in the excited state was formed. Then one of the two possible modes was realized:

1) The compound-nucleus could lose its excitation and formed a stable nucleus being heavier than Pd one;

2) The compound nucleus could be fissionable into two nuclei-fragments with masses less than Pd. In so doing the two nuclei should be in the excited isomer state. (Experiments showed that the nuclear reaction energy was not produced as kinetic energy).

6 - Determining the specific physical mechanism of these reactions will require additional research. One of the possible types of these reactions that form anomalous nuclides may be a long-range (resonant) nuclear reaction. The mechanism of such long-range reactions can be considered by the example of the specific transmutation reaction (Fig. 13). The formation of many ${ }^{13} \mathrm{C}$ nuclides was recorded in these experiments. A possible reaction can be the following.

${ }^{104} \mathrm{Pd}+{ }^{2} \mathrm{D} \rightarrow{ }^{106}[\mathrm{Pd} ; \mathrm{D}]^{*} \rightarrow{ }^{13} \mathrm{C}+{ }^{93} \mathrm{Nb}+7.820 \mathrm{MeV} ;(5)$

According to the laws of pulse and energy conservation, the formed nuclide ${ }^{13} \mathrm{C}$ should receive the energy of $6.8608 \mathrm{MeV}$. The nuclide ${ }^{93} \mathrm{Nb}$ should receive the energy of $0.959 \mathrm{MeV}$. The nuclear excited state (a nuclear isomer) with the energy of $6.864 \mathrm{MeV}$ and excited level width of $6 \mathrm{keV}$ existed for ${ }^{13} \mathrm{C}$.The excited level with the energy of $0.9498 \mathrm{MeV}$ existed for ${ }^{93} \mathrm{Nb}$. The difference between the energy received by nuclide ${ }^{13} \mathrm{C}$ and the energy of one of the excited nucleus state was equal $3.2 \mathrm{keV}$. At the excitation energy of the crystalline lattice of 1.5 and the width of the excited energy level of 6.0, these conditions gave a high probability of carrying out the long-range (resonant) nuclear reaction (Fig. 13).

The totality of the experimental results supports the assumption that the energy of excited nuclear levels of the formed nuclides converts into heat. The specific physical mechanism of such conversion requires additional research. 


\section{CONCLUSIONS}

The obtained results - excess heat up to $5 \mathrm{~W} / \mathrm{cm}^{2}$ at the efficiency up to $150 \%$ - should allow the development of a demonstration heater. The technology of multi-element cathode fuel elements with plasma anodes has been developed. New nuclear engineering is possible, based on non-equilibrium nuclear transmutation reactions in solid-state medium. This type of engineering can be called the "Third way" in nuclear engineering, in comparison with the nuclear engineering on the basis of uranium fission and thermonuclear fusion.

\section{REFERENCES}

1. A. B. Karabut, "Excess Heat Power, Nuclear Products and X-ray Emission in Relation to the High Current Glow Discharge Experimental Parameters", Proceedings of the $9^{\text {th }}$ International Conference on Cold Fusion, May 19-24, 2002, China, p.151.

2. A. B. Karabut, Patent No 2240612 RU, "Method of Heat Energy Production", Russia.

\section{FIGURES}

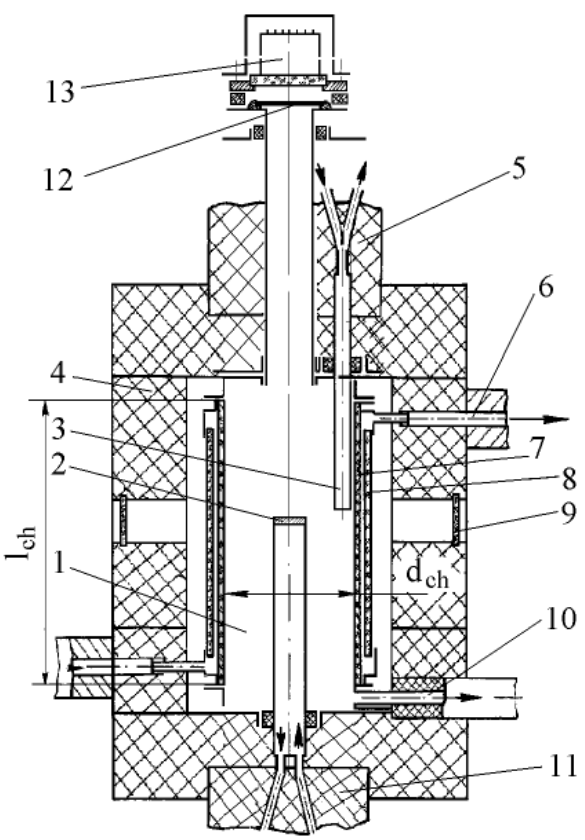

Fig. 1. Experimental Glow Discharge Device (flow continuous calorimeter). 1 - vacuum discharge chamber, 2 -cathode unit. 3 -anode unit, 4 -thermal insulation cover, 5 -insulation of the anode cooling system, 6 - the chamber cooling system, 7 - the discharge chamber tube, 8 - the chamber cooling jacket tube, 9 - windows in thermal insulation cover, 10 - the vacuum hose, 11 - insulation of the cathode cooling system. 12 - Be window, 13 - X-ray detector. 

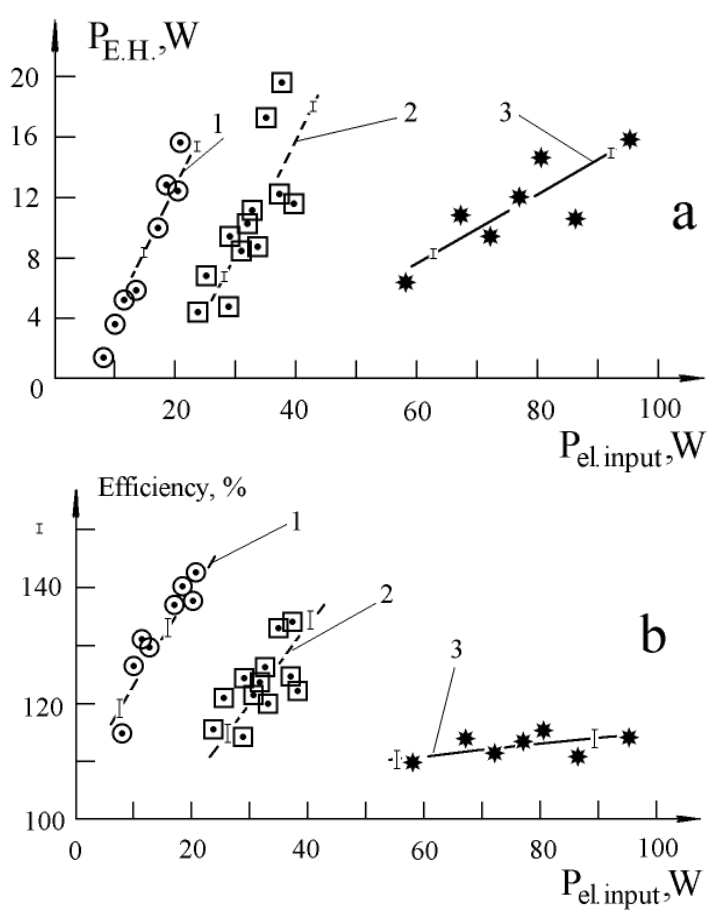

Fig. 2. Excess heat (a) and Efficiency (b) in relation to input electric power. Cathode sample - Pd, $\mathrm{d}=9 \mathrm{~mm}$, gas - $\mathrm{D}_{2} ; 1-\mathrm{j}=12 \mathrm{~mA} / \mathrm{cm}^{2}, 2-22 \mathrm{~mA} / \mathrm{cm}^{2}, 3-70 \mathrm{~mA} / \mathrm{cm}^{2}$. 

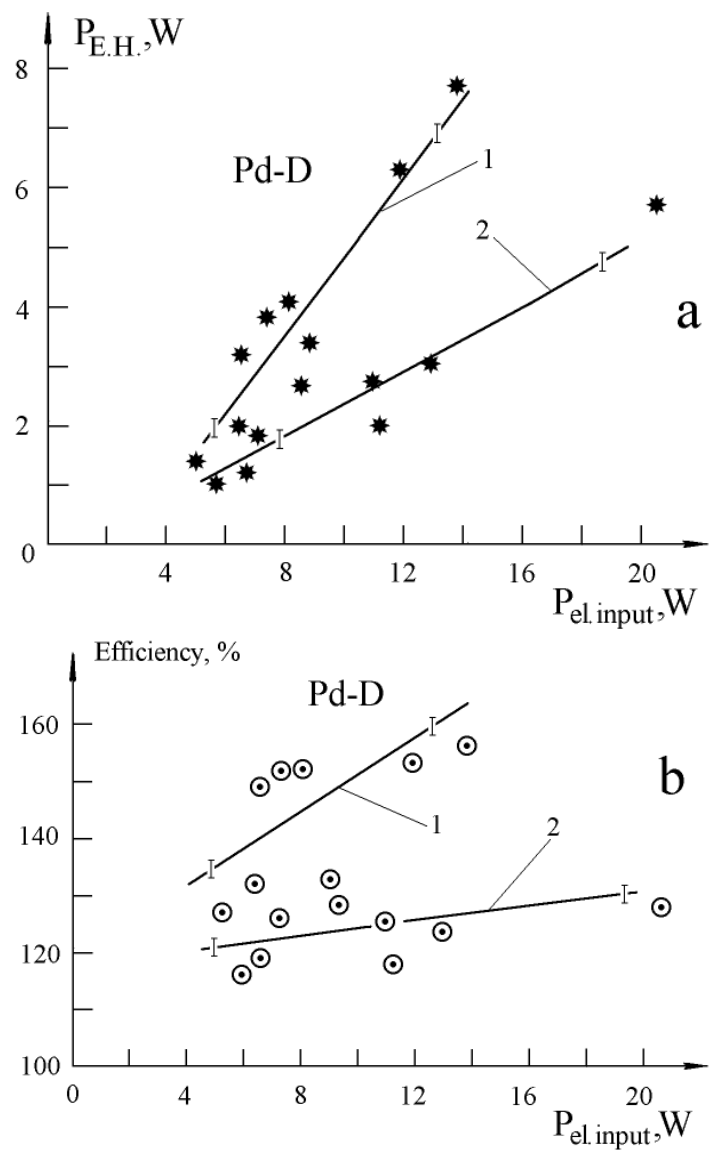

Fig. 3. Excess heat (a) and Efficiency (b) in relation to input electric power. Cathode sample - Pd, $d=9 \mathrm{~mm}$, gas - $\mathrm{D}_{2} ; 1-\mathrm{D}_{2}$-charged Pd with $\mathrm{D}_{2}$ loading and unloading, 2 - $\mathrm{D}_{2}$-charged Pd without $\mathrm{D}_{2}$ loading or unloading 

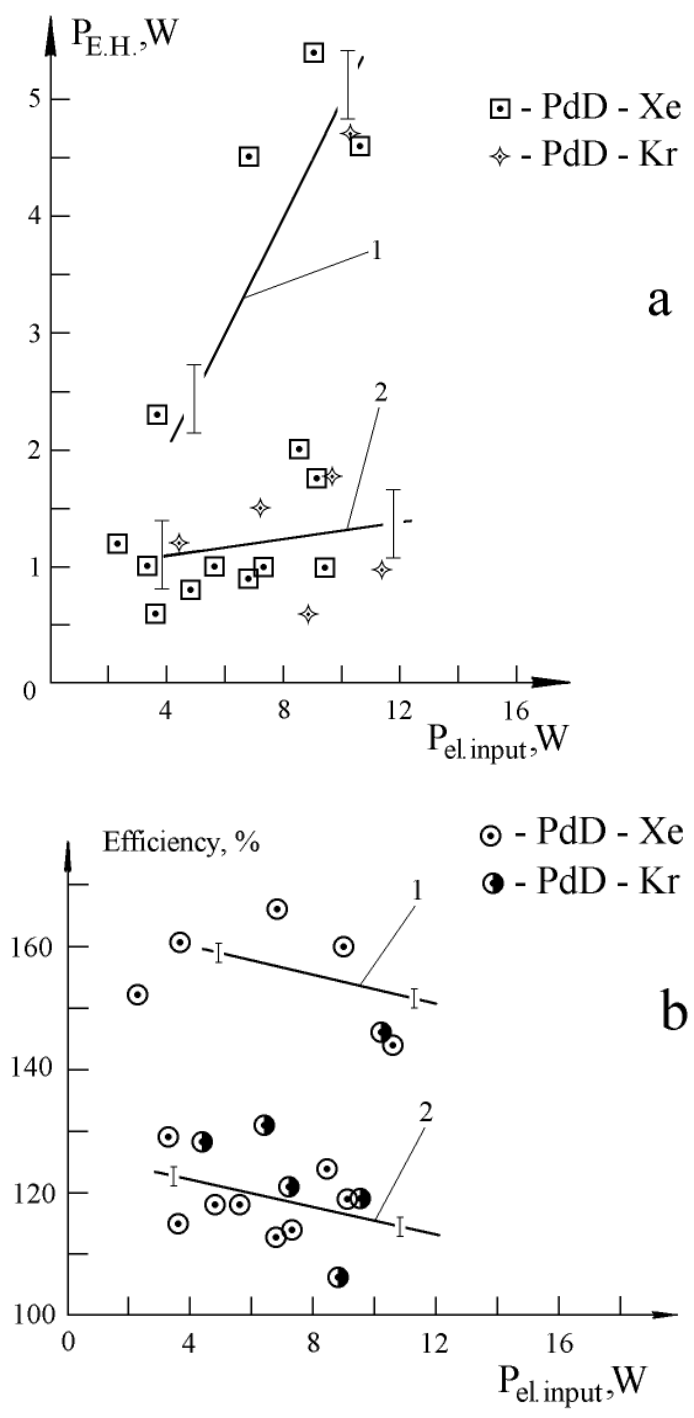

Fig. 4. Excess heat (a) and Efficiency (b) in relation to the input electric power. Deuterium-charged Pd cathode samples in Xe and Kr discharges, $d=9$ mm. 1- $D_{2}$-charged Pd with $D_{2}$ loading and unloading, 2- $D_{2}-$ charged Pd without $\mathrm{D}_{2}$ loading or unloading.

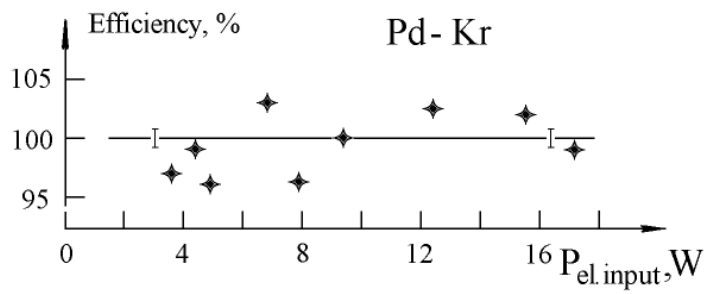

Fig. 5. Efficiency in relation to the input electric power. Not deuterium-charged Pd cathode samples in $\mathrm{Kr}$ discharges, $\mathrm{d}=9 \mathrm{~mm}$. 


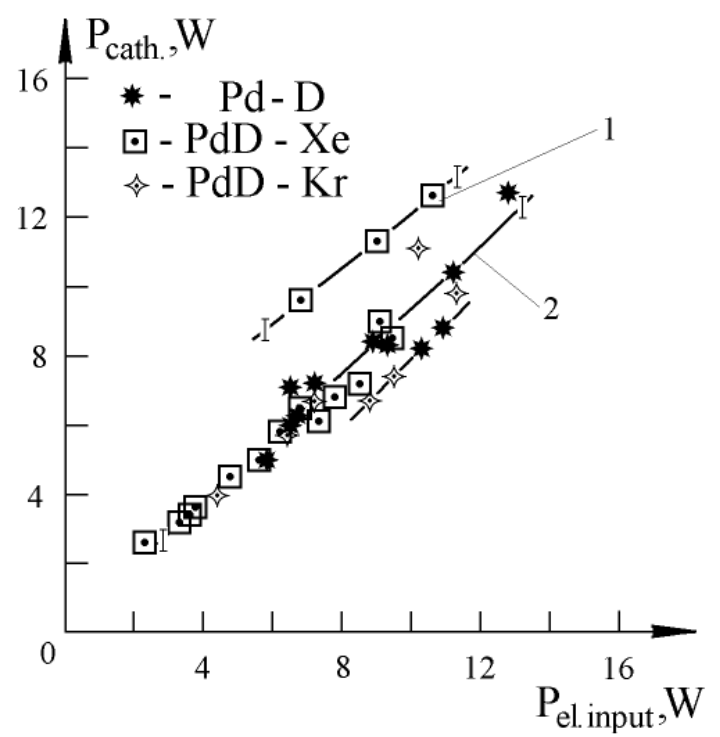

Fig. 6. Excess heat (a) and Efficiency (b) in relation to input electric power for a Pd cathode in D and deuterium-charged Pd cathode samples in Xe and Kr discharges. 1,2 - see Fig. 3. and Fig. 4. 1- $\mathrm{D}_{2}$-charged Pd with $\mathrm{D}_{2}$ loading and unloading, 2- $\mathrm{D}_{2}$-charged $\mathrm{Pd}$ without $\mathrm{D}_{2}$ loading or unloading.

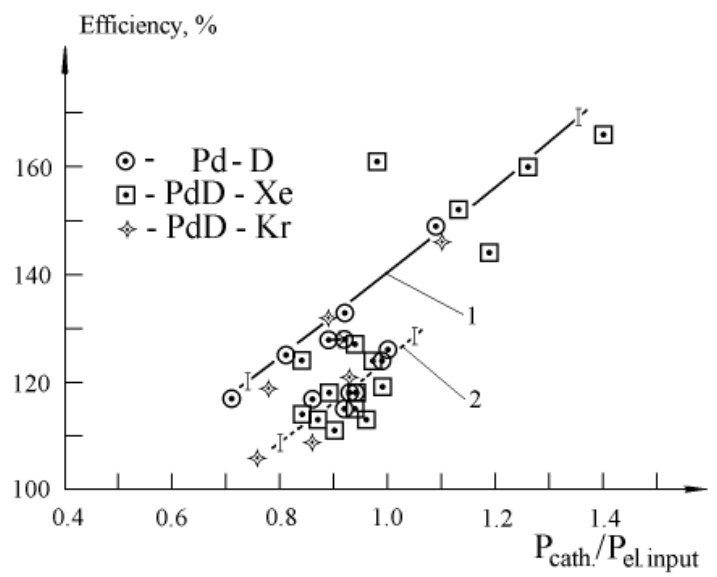

Fig. 7. Dependence of excess heat and efficiency on parameters: relation of heat power released on cathode to electrical power input into discharge. $1-\mathrm{D}_{2}$-charged Pd with $\mathrm{D}_{2}$ loading and unloading, 2- $\mathrm{D}_{2}$-charged $\mathrm{Pd}$ without $\mathrm{D}_{2}$ loading or unloading. 


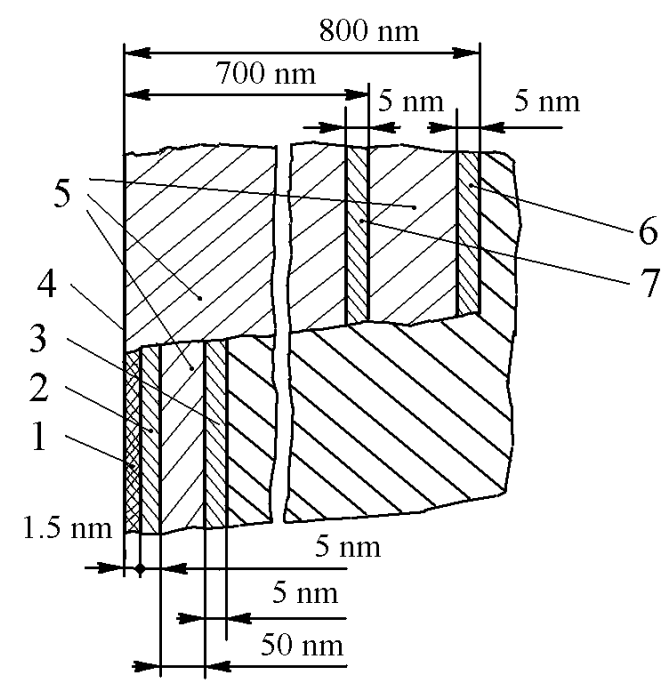

Fig. 8. Registration procedure for the impurity contents in the cathode samples (methods SIMS and SNMS). 1-dirty superficial layer, 2,3-analyzed layers, 4-surface of the cathode samples, 5-removal of a metal layer, 7,6- analyzed layers.

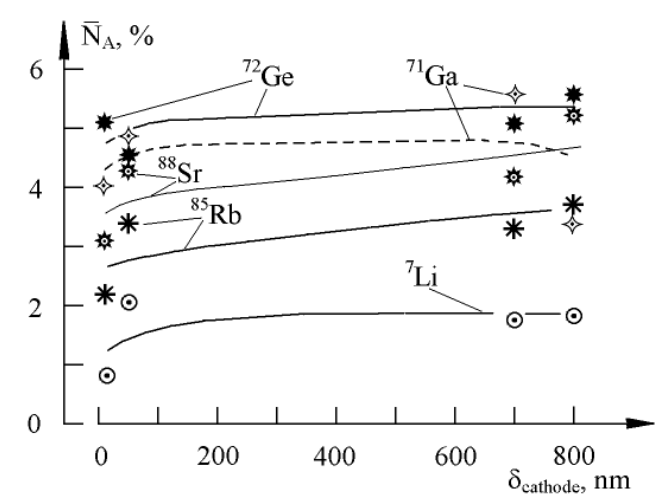

Fig. 9. Variation of some isotopes in the surface layer Pd - the cathode after discharge, System Pd - D2, current $-100 \mathrm{~mA}$, time of the experiment -22 hours. 


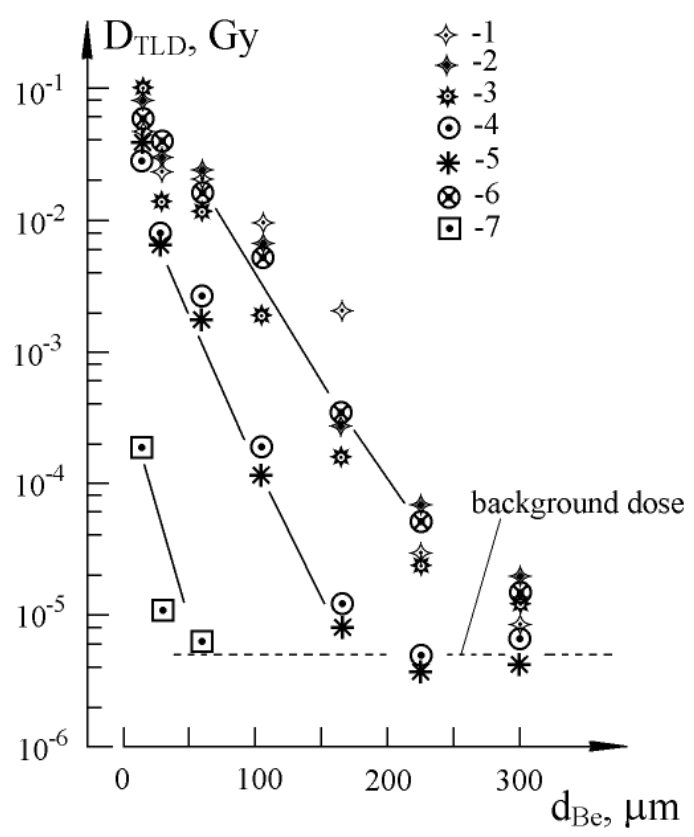

Fig. 10. The X-ray dose absorbed by TLD detectors covered with Be foil with the different thickness. Pd-D system, current $-200 \mathrm{~mA}$, the exposure time -6000s. 1 - discharge voltage is $1750 \mathrm{~V}, 2-1770 \mathrm{~V}, 3-1650 \mathrm{~V}, 4$ 1530V, 5 - 1400V, 6 - 1250V, 7 - 800V.

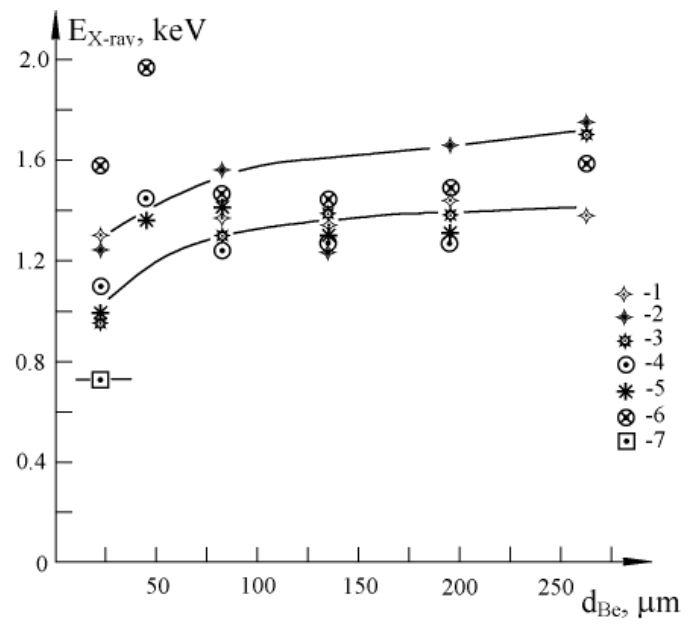

Fig. 11. The X-ray emission energy dependence upon the discharge voltage (TLD detectors with Be foil shields measurement ). 1 - discharge voltage is $1750 \mathrm{~V}, 2-1770 \mathrm{~V}, 3-1650 \mathrm{~V}, 4-1530 \mathrm{~V}, 5-1400 \mathrm{~V}, 6$ $1250 \mathrm{~V}, 7$ - 800V. 


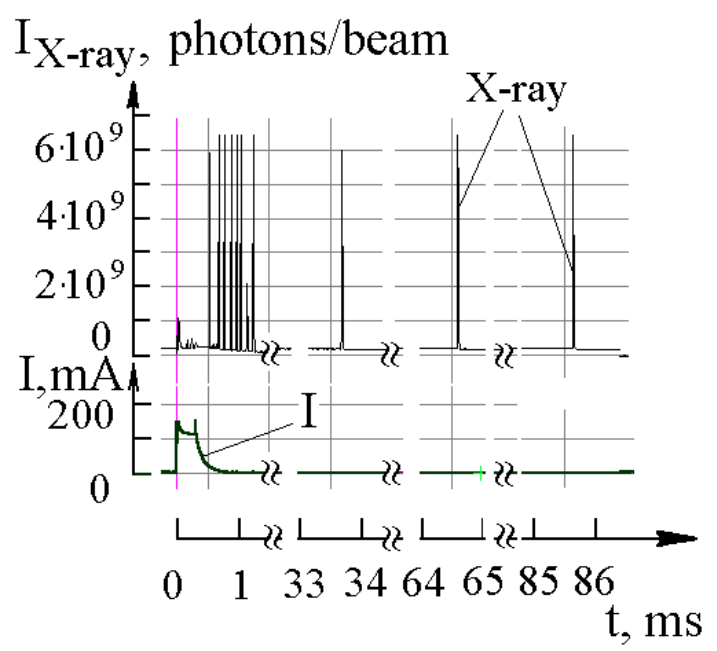

Fig. 12. Typical oscillogram of X-ray bursts within long time interval after turning off the discharge current.

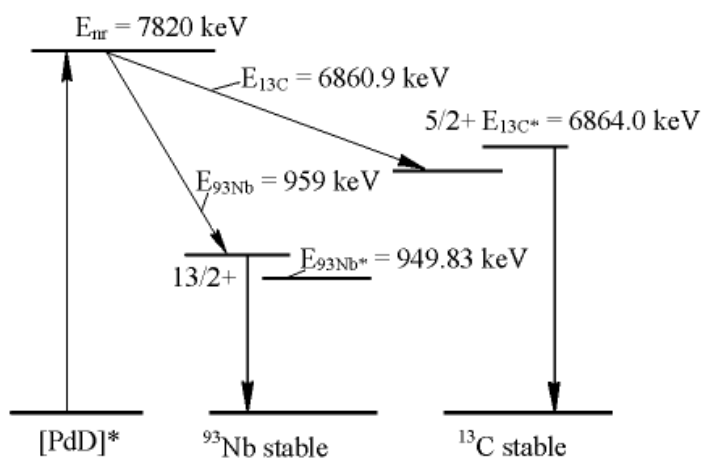

Fig. 13. Assumed pathways for long-range (resonant) nuclear reactions. 